\title{
Abnormal cutaneous flexor reflex activity during controlled isometric plantarflexion in human spinal cord injury spasticity syndrome
}

\author{
J Gómez-Soriano ${ }^{1,2}$, E Bravo-Esteban ${ }^{3,4}$, E Pérez-Rizo5 ${ }^{5}$ G Ávila-Martín², I Galán-Arriero², C Simón-Martinez ${ }^{2}$ \\ and J Taylor ${ }^{2,6,7}$
}

Study design: Although abnormal cutaneous reflex (CR) activity has been identified during gait after incomplete spinal cord injury $(\mathrm{SCl})$, this activity has not been directly compared in subjects with and without the spasticity syndrome.

Objectives: Characterisation of CR activity during controlled rest and 'ramp and hold' phases of controlled plantarflexion in subjects with and without the $\mathrm{SCl}$ spasticity syndrome.

Design: Transverse descriptive study with non-parametric group analysis.

Setting: SCl rehabilitation hospital.

Methods: Tibialis Anterior (TA) reflexes were evoked by innocuous cutaneous plantar sole stimulation during rest and ramp and hold phases of plantarflexion torque in non-injured subjects $(n=10)$ and after $\mathrm{SCl}$ with $(n=9)$ and without $(n=10)$ hypertonia and/or involuntary spasm activity. Integrated TA reflex responses were analysed as total (50-300 ms) or short (50-200 ms) and long-latency (200-300 ms) activity.

Results: Total and long-latency TA activity was inhibited in non-injured subjects and the $\mathrm{SCl}$ group without the spasticity syndrome during plantarflexion torque but not in the $\mathrm{SCl}$ spasticity group. Furthermore, loss of TA reflex inhibition during plantarflexion correlated with time after $\mathrm{SCl}(\rho=0.79, P=0.009)$. Moreover, TA reflex activity inversely correlated with maximum plantarflexion torque in the spasticity group $(\rho=-0.75, P=0.02)$, despite similar non-reflex TA electromyographic activity during plantarflexion after $\mathrm{SCl}$ in subjects with $(0.11,0.08-0.13 \mathrm{mV})$ or without the spasticity syndrome $(0.09,0.07-0.12 \mathrm{mV})$.

Conclusions: This reflex testing procedure supports previously published evidence for abnormal CR activity after $\mathrm{SCl}$ and may characterise the progressive disinhibition of TA reflex activity during controlled plantarflexion in subjects diagnosed with the spasticity syndrome.

Spinal Cord (2016) 54, 687-694; doi:10.1038/sc.2016.9; published online 23 February 2016

\section{INTRODUCTION}

Spasticity was originally defined as an increase in velocity-dependent, tonic stretch reflexes to passive movement ${ }^{1}$ and has been used to describe a number of signs and symptoms that together contribute to the syndrome. ${ }^{2}$ Cutaneous reflex (CR) dysfunction has also been regarded as an additional sign of the spasticity syndrome following spinal cord injury (SCI), ${ }^{3-9}$ especially when detected in subjects with hypertonia and increased tonic stretch reflexes. ${ }^{10-12}$ In addition, abnormal flexor reflex excitability is present during subacute ${ }^{4,13}$ and chronic SCI, ${ }^{14,15}$ impacts on residual gait function after $\mathrm{SCI}^{16}$ and interferes with daily activities. ${ }^{17}$

Lower limb CR activity in humans is modulated by several segmental and descending control mechanisms, ${ }^{18-21}$ and the loss of descending modulatory mechanisms may contribute to the SCI spasticity syndrome. Tibialis Anterior (TA) muscle reflex activity evoked following cutaneous stimulation of the plantar surface $(\mathrm{Pl}-\mathrm{TA} \mathrm{CR})^{22-24}$ has been used as a test to assess the integrity of segmental and descending motor control mechanisms in healthy subjects with physiological reflex modulation during the step-cycle in healthy subjects. ${ }^{3}$ Typically, the TA CR is strongly inhibited during the stance phase, ${ }^{25}$ whereas this inhibition is reduced naturally during the swing phase of gait. ${ }^{3,11,26,27}$ Following incomplete SCI, inhibitory modulation of CR activity during gait is partially lost in subjects diagnosed with the SCI spasticity syndrome, ${ }^{11}$ although this observation was not controlled by comparison with a SCI group without spasticity. In contrast, abnormal CR activity observed during rhythmic ankle joint displacement in subjects with spasticity ${ }^{3,10}$ strongly supports systematic flexor reflex testing during rest and controlled plantarflexion. ${ }^{28}$ The hypothesis of this study, therefore, is that testing for abnormal CR function during both rest and controlled ramp and hold phases of controlled plantarflexion after incomplete SCI would demonstrate better loss of physiological inhibitory control, specifically in subjects with the spasticity syndrome, including any potential negative impact on muscle paresis. ${ }^{29-31}$

${ }^{1}$ Toledo Physiotherapy Research Group (GIFTO), Nursing and Physiotherapy School, Castilla La Mancha University, Toledo, Spain; ${ }^{2}$ Sensorimotor Function Group, Hospital Nacional de Parapléjicos, SESCAM, Toledo, Spain; ${ }^{3}$ Neural Rehabilitation Group, Instituto Cajal, Council for Scientific Research (CSIC), Madrid, Spain; ${ }^{4}$ Physiotherapy Faculty, Zaragoza University, Zaragoza, Spain; ${ }^{5}$ Biomechanics Unit, Hospital Nacional de Parapléjicos, SESCAM, Toledo, Spain; ${ }^{6}$ Stoke Mandeville Spinal Research, National Spinal Injuries Centre, Buckinghamshire Healthcare Trust, NHS, Aylesbury, UK and ${ }^{7}$ Harris Manchester College, Oxford University, Oxford, UK

Correspondence: Dr J Taylor, Sensorimotor Function Group, Hospital Nacional de Parapléjicos, SESCAM, Finca "La Peraleda" s/n, Toledo 45071, Spain.

E-mail: jscott@sescam.org or julian.taylor@smsr.org

Received 9 July 2015; Received 18 December 2015; accepted 2 January 2016; published online 23 February 2016 
The objective of this study was to characterise abnormal TA CR activity measured during controlled plantarflexion in subjects with and without the spasticity syndrome. In this study, we show that abnormal $\mathrm{CR}$ activity measured during controlled ramp and hold plantarflexion in subjects with the SCI spasticity syndrome suggests damage to the descending modulatory mechanisms and that this activity may develop during subacute and chronic phase of injury. ${ }^{4}$

\section{MATERIALS AND METHODS}

\section{Subjects and general procedures}

The experimental protocol was approved by the Hospital's Clinical Research Ethics Committee, and signed consent was obtained from the subjects in accordance with the Helsinki Declaration of 1975. Sixteen non-injured subjects (10 women and 6 men) with no central or peripheral neurological deficit and 19 individuals with SCI were recruited. The inclusion criteria for individuals with SCI were as follows: age range 18-65 years; motor incomplete SCI, diagnosed as grade C or D using the ASIA Impairment Scale (AIS); ${ }^{32}$ a neurological level of SCI between C4 and Th10; SCI greater than 3 months time post injury; and preservation of muscle function for both the TA and Triceps Surae (TS) scored with at least 3 on the International Muscle Grading Scale. ${ }^{33}$ The exclusion criteria included the following: peripheral nerve injury (that is, no denervation), which may have influenced reflex function in subjects with lower thoracic SCI or neurotrauma at the supraspinal level corroborated with a neurological examination; a clinical history of epilepsy; pregnancy; lower limb joint and/or muscle injuries; and failure to elicit reflex responses in the TA muscle following non-noxious electrical stimulation of the plantar pad at sensory threshold for observable CR activity applied at a tolerable intensity (see below).

For the study purposes, SCI spasticity syndrome was defined by the presence of spastic hypertonia assessed using the modified Ashworth scale ${ }^{34}$ and involuntary muscle activity assessed with the Penn spasm frequency scale. ${ }^{35}$ Following evaluation, subjects were assigned to the SCI spasticity group $(n=9)$ when the lower limb modified Ashworth scale scored higher than 1 and/or a Penn spasm score was rated higher than 0 . As considered in previous studies, slight muscle hypertonia ( 1 in the modified Ashworth scale) without the presence of spasms ( 0 in the Penn scale) was operationally defined as not characteristic of the spasticity syndrome. ${ }^{16,36,37}$ Ten subjects with SCI were recruited without the spasticity syndrome. All clinical evaluations were assessed by a physiotherapist working at the hospital who was blinded to the experimental design of the study. Patients followed their standard treatment for spasticity, which included in some cases oral baclofen.

\section{Controlled isometric ramp and hold plantarflexion torque}

Subjects were seated with their hip, knee and ankle joints set at an angle of $90^{\circ}$ and their foot placed on a custom-built dynamometer (Figure 1a) that offered visual feedback for plantarflexion torque (Figure 1b). ${ }^{37}$ The foot was secured in place with Velcro straps immediately following placement of the plantar electrode. The entire dynamometer platform acted to measure plantarflexion torque (Figures 1a and c). The task was performed with the dominant leg in the case of the non-injured control subjects or with the limb with the higher muscle score for the SCI subjects. ${ }^{33}$ Maximum voluntary torque (MVT) was recorded in all participants during isometric plantarflexion hold and was calculated as the average of three maximum 5 -s contractions separated by 20 -s intervals. Subjects were instructed to follow a visual feedback-guided plantarflexion pattern that included ramp and hold isometric contractions (Figure 1b). Subjects were familiarised with the task at least one time before recording. The target template was programmed to present 10 plantarflexion ramp and hold targets on a monitor, which the subject was asked to match by performing controlled ramp and hold isometric activation of the TS muscle at $50 \%$ MVT. The template provided an audible warning if the plantarflexion torque recorded during either the ramp or the hold phase deviated more than $10 \%$ from the target torque. The initial 3-s rest period of the template was set at $5 \%$ maximum isometric plantarflexion torque value, which was equivalent to the weight of the foot on the dynamometer without evident background electromyographic (EMG) activity from the TS. The motor task was initiated with 4-s isometric ramp phase, to reach the $50 \%$ MVT, and a 4 -s isometric hold phase, keeping the 50\% MVT of plantarflexion, followed by a 4-s relaxation period. Plantarflexion torque and Pl-TA CR activity data were collected during the controlled motor task, converted to digital form and analysed using custom-built software (LabView Version 7, MicroPlus 1401 and Signal version 2.14).

\section{Pl-TA CR activity measured during ramp and hold plantarflexion torque}

The plantarflexion protocol was first performed without evoked CR activity to record EMG that coincided with the same period of reflex analysis performed during controlled plantarflexion. Pl-TA CR activity was then evoked during the rest, ramp and hold phases of the controlled isometric plantarflexion (Figure $1 \mathrm{~b}, 10$ trials for each TS contraction). Pl-TA CR activity was evoked $2 \mathrm{~s}$ into the rest, ramp and hold phases of controlled plantarflexion torque. The anode $(8 \times 10 \mathrm{~cm})$ was placed on the dorsal surface of the foot and the cathode $(4 \times 6 \mathrm{~cm})$ was positioned on the plantar sole between the first and the second metatarsal to permit localised cutaneous stimulation. Testing within the laboratory confirmed that this form of reflex stimulation was comfortable when compared with electrical stimulation applied to the skin overlying the sural or tibial nerve.

Special care was also taken to apply stimuli that were not noxious, at an intensity close to reflex threshold, and that were perceived within the plantar sole following incomplete SCI. Reflex activity was evoked with a constant current stimulator (DS7A, Digitimer Ltd., Welwyn Garden City, Hertfordshire, UK), which applied electrical stimulation delivered as five rectangular pulses (1 ms duration with a 5-ms interval). ${ }^{38}$ TA CR threshold was defined during the rest period (Figure 1a) as the non-painful stimulus intensity sufficient to elicit an observable Pl-TA CR response. The TA CR threshold was identified with test stimuli applied every $20 \mathrm{~s}$. Thereafter, the plantar stimulus intensity was set as a 1.2 multiple of the individual Pl-TA reflex threshold.

\section{CR measurement and analysis}

Background signal and CR EMG activity were measured from the TA, in addition to non-reflex activity recorded from the Gastrocnemius Medialis muscle, with bipolar silver chloride electrodes $(1000 \times$ amplification $)$ with a built-in $20-450 \mathrm{~Hz}$ bandpass filter (Signal Conditioning Electrodes version 2.3). EMG activity was sampled at $10 \mathrm{KHz}$ (MicroPlus 1401), whereupon reflex activity was full-wave rectified (Signal version 2.14). Integration of total reflex activity (50-300 ms) was performed as the primary outcome measure in subjects with SCI with $(n=9)$ or without the spasticity syndrome $(n=10$, Figure 1c), which was consistent with previous studies. ${ }^{11,38}$ However, specific CR activity was also calculated for short (50-100 ms), medium (100-200 ms) and long latency (200-300 ms) in Table 2, to broadly differentiate long-latency reflex activity. ${ }^{14}$ Inclusion of the SCI group without diagnosis of the spasticity syndrome was performed to incorporate an appropriate control group for comparison of $\mathrm{CR}$ activity. Pl-TA reflex examples were presented following a 3-point smoothing function for clarity in Figure 1c.

Integrated Pl-TA reflex activity was normalised with respect to the rest period using a technique adopted in other reflex studies. ${ }^{11,38,39}$ First, background EMG signal activity was subtracted from evoked TA reflex activity. Second, reflex activity ratios were calculated between the ramp or hold phases of plantarflexion and activity measured during the rest period. Finally, the value of 1 was subtracted from the ratio to obtain normalised values of reflex modulation during movement. Hence, CR activity measured during the rest phase was expressed as a zero score, in contrast to negative values calculated during controlled plantarflexion, which represented reflex inhibition.

\section{Statistical analysis}

Normality, descriptive and inferential statistical tests were performed with a commercial statistical software package (Prism version 4.0). Normality of the reflex-data distribution was tested with the Kolmogorov-Smirnov procedure using a Lilliefors significance level, and the data were described as non-Gaussian. Hence, non-parametric statistical analysis and data presentation were justified.

Inter-group comparisons were performed using the Kruskall-Wallis test with a post hoc Bonferroni correction. Background EMG and evoked CR activity data normalised to the rest period were statistically analysed using the Friedman test 
a

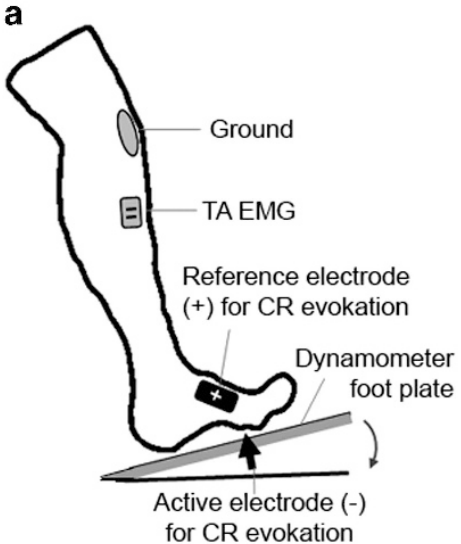

C
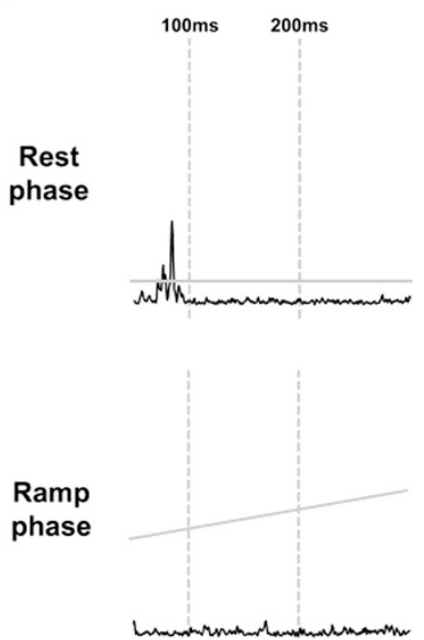

\begin{tabular}{l|l|l} 
Hold \\
phase
\end{tabular}

NON-INJURED CONTROLS b

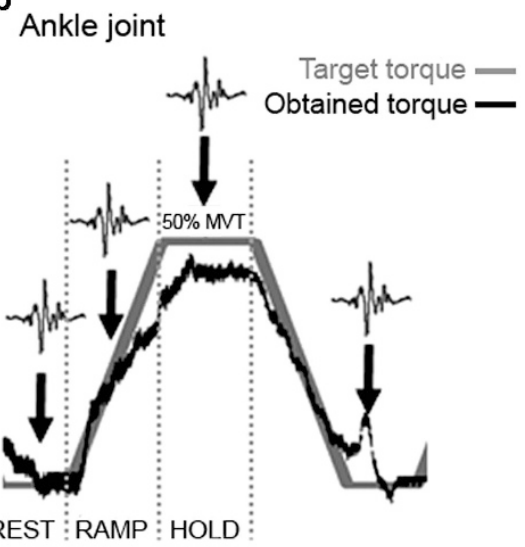

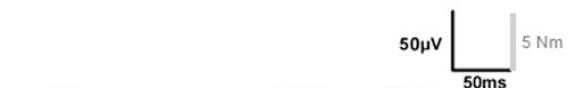
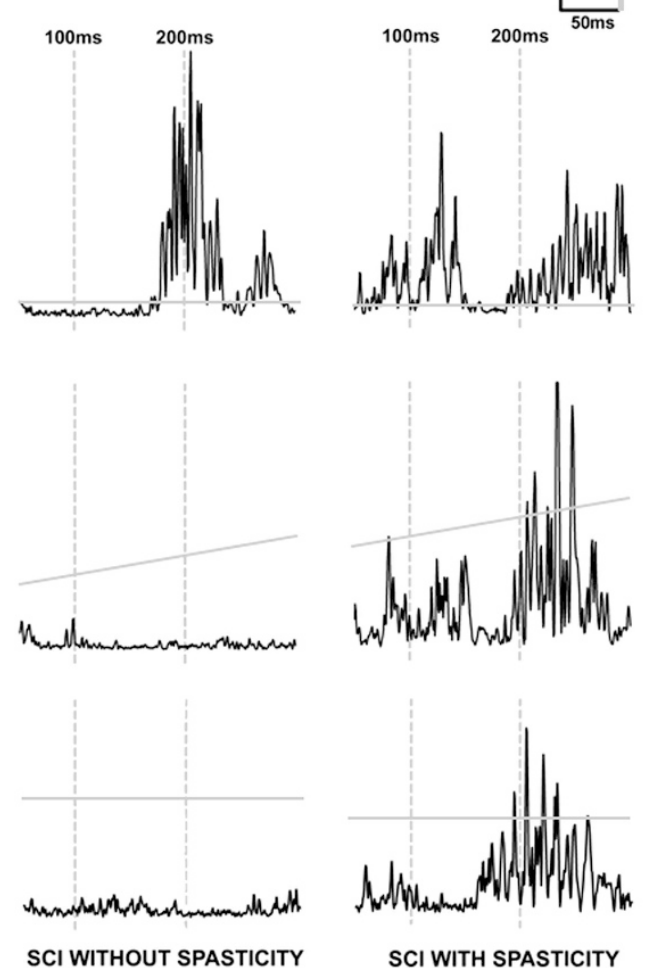

Figure 1 Plantar-TA reflex testing method, motor task template and reflex EMG records recorded during rest and controlled ramp and hold phases of plantarflexion torque. (a) Schematic diagram illustrating the placement of the electrical stimulating plantar and TA EMG electrode used to evoke and record CR activity. (b) TA CR activity was evoked at rest (5\% of MVT recorded with the dynamometer) and during the ramp and hold phase of plantarflexion torque (set at 50\% MVT). (c) PI-TA CR recorded from a non-injured healthy subject (left column), an individual with SCl without (centre column) and a person diagnosed with the SCl spasticity syndrome (right column), during the rest, ramp and hold phases of plantarflexion. Early (50-100 ms), medium (100-200 ms) and long-latency (200-300 ms) PI-TA CR activity are shown for all rectified EMG records, with the majority of reflex activity measured within the long-latency window for the $\mathrm{SCl}$ groups. Note that $\mathrm{CR}$ activity up to $50 \mathrm{~ms}$ is not presented and that background TA EMG was not significantly different between the experimental groups (see results section).

and post hoc Bonferroni correction. Correlation analysis was performed with Spearman's rank test to detect relationships between the CR responses, plantarflexion MVT and standard measures of the spasticity syndrome. ${ }^{4-8}$ EMG, CR and functional measures were expressed as the median with the 25th-75th percentile values. The null hypothesis was rejected at $P<0.05$.

\section{RESULTS}

In general, similar demographic, clinical or functional characteristics were observed between the SCI groups with and without spasticity (Table 1). The age of the SCI groups was also similar (Table 1), although a significant difference was identified for the younger university students $(23.6 \pm 6.8$, mean \pm s.e. $)$ enroled in the non-injured group compared with all subjects with SCI $(44.5 \pm 14.6$, $P<0.01$, Mann-Whitney test). No differences in gender were identified between non-injured controls and subjects with SCI.

Pl-TA CR modulation during controlled isometric plantarflexion Typical individual Pl-TA CR reflex records are presented in Figure 1c for both non-injured subjects (left column) and SCI subjects without (middle column) and with spasticity (right column) during the rest 
period and both the ramp and hold phases of controlled isometric plantarflexion. Pl-TA CR latency and duration time for the SCI group without spasticity syndrome were $173 \pm 12 \mathrm{~ms}$ and $74 \pm 13 \mathrm{~ms}$, compared with $154 \pm 19 \mathrm{~ms}$ and $138 \pm 20 \mathrm{~ms}(P<0.05)$ for the group with spasticity. The majority of the total Pl-TA CR activity was detected from $100 \mathrm{~ms}$ for both the SCI groups (Figure 1c). Closer examination of the CR records indicated the inhibition of total Pl-TA reflex during the ramp and hold phases of plantarflexion torque in the non-injured and SCI subject without the spasticity syndrome. In contrast, no inhibition of Pl-TA CR activity was observed during plantarflexion torque in the subject diagnosed with the SCI spasticity syndrome (Figure 1c).

Table 1 Demographic and neurological characteristics of subjects with $\mathrm{SCl}$ spasticity

\begin{tabular}{lcc}
\hline & $\begin{array}{c}\text { SCI without spasticity } \\
(\mathrm{n}=10)\end{array}$ & $\begin{array}{c}S C l \text { without spasticity } \\
(\mathrm{n}=9)\end{array}$ \\
\hline Age (years) & $51 \pm 12$ & $37 \pm 14$ \\
Gender & $8 \mathrm{Male}$ & $8 \mathrm{Male}$ \\
Aetiology & 9 Trauma/1 Medical & 7 Trauma/2 Medical \\
AIS score (A, B, C, D) & $4 \mathrm{C} / 6 \mathrm{D}$ & $7 \mathrm{C} / 2 \mathrm{D}$ \\
Neur. level SCI & $9 \mathrm{C} / 1 \mathrm{Th}$ & $6 \mathrm{C} / 3 \mathrm{Th}$ \\
Time from SCl (months) & $7 \pm 4$ & $6 \pm 2$ \\
TA muscle score (0-5) & $4(3-4)$ & $4(3-4)$ \\
TS muscle score (0-5) & $4(4-4)$ & $4(4-4)$ \\
Max. vol. Pl. Tor. (Nm) & $21 \pm 8$ & $19 \pm 8$ \\
Modified Ashworth score & $0(0.0-0.0)$ & $\mathbf{2 . 0}(\mathbf{1 . 0}-\mathbf{3 . 0})^{\mathrm{a}}$ \\
(0-4) & & $\mathbf{2 . 0}(\mathbf{1 . 8}-\mathbf{2 . 0})^{\mathrm{a}}$ \\
PENN score (0-4) & $0(0.0-0.0)$ & \\
\hline
\end{tabular}

Abbreviations: AIS, ASIA impairment scale; C, cervical; Neur. Level, neurological level; Max. PI. Tor., maximum plantarflexion torque; SCl, spinal cord injury; TA, Tibialis Anterior; Th, Thoracic; TS, Triceps Surae. All values presented as mean \pm s.e., except muscle score, modified Ashworth scale and Penn scores, which are presented as median values with 25 th-75th percentile range. asignificant difference $(P<0.001)$ when compared with the group without spasticity

(Mann-Whitney Test). Statistical significant data are represented in bold.
Median integrated Pl-TA CR activity was similar between the experimental groups when analysed within the early (50-100 ms) or medium (100-200 ms) latency window during the rest period or ramp and hold phases of controlled isometric plantarflexion (Table 2). Long-latency Pl-TA CR activity was higher for the SCI spasticity syndrome group when compared with the early reflex response in the non-injured control group during rest (Table 2). Furthermore, during controlled isometric plantarflexion, the $200-300 \mathrm{~ms}$ Pl-TA CR activity was higher in the SCI spasticity group compared with the SCI group without spasticity (Table 2). Reflex stimulation intensities were similar when compared between non-injured subjects 29 (25-45) $\mathrm{mA}$, the group without SCI spasticity $38(34-55) \mathrm{mA}$ and the group with SCI spasticity $40(28-50) \mathrm{mA}(P=0.30)$.

Pl-TA reflex activity measured during the rest period and during either the ramp or hold phase of plantarflexion torque is presented in Table 3. Subjects with SCI spasticity syndrome showed higher activity during both the ramp and hold phases of plantarflexion but not during rest.

Pl-TA CR activity analysed following the subtraction of background EMG activity and normalised to the rest period is shown in Table 4. As shown previously, the normalised reflex data demonstrated that both the non-injured controls and SCI group without the spasticity syndrome revealed Pl-TA CR inhibition during plantarflexion. In contrast, a loss of physiological inhibition of reflex function during controlled isometric plantarflexion was observed in the SCI group diagnosed with the spasticity syndrome (Table 4).

Pl-TA CR activity, plantarflexor torque and spasticity measures Pl-TA CR amplitude correlated negatively with residual plantarflexion torque during ramp plantarflexion $(\rho=-0.75, P=0.016$, Figure $2 \mathrm{a})$ in the SCI spasticity syndrome group, although this was not evident in either the the rest period $(\rho=-0.28, P=0.43)$ or isometric hold phase of plantarflexion torque $(\rho=-0.54, P=0.11)$. The possibility that increased TA muscle activation contributed to plantarflexor weakness in the SCI spasticity group was controlled following identification that

Table 2 Short, medium and long-latency integrated PI-TA CR activity measured from non-injured individuals and from subjects with spinal cord injury with and without spasticity

\begin{tabular}{|c|c|c|c|}
\hline & $\begin{array}{c}\text { 50-100 ms } \\
\text { PI-TA CR } \\
\text { activity } \\
\text { (mV.s) }\end{array}$ & $\begin{array}{c}\text { 100-200 ms } \\
\text { PI-TA CR } \\
\text { activity } \\
\text { ( mV.s) }\end{array}$ & $\begin{array}{c}\text { 200-300 ms } \\
\text { PI-TA CR } \\
\text { activity } \\
\text { (mV.s) }\end{array}$ \\
\hline \multicolumn{4}{|l|}{ Rest } \\
\hline Non-injured controls & $0.06(0.04-0.07)$ & $0.08(0.04-0.10)$ & $0.09(0.04-0.12)$ \\
\hline \multicolumn{4}{|l|}{ Ramp } \\
\hline Non-injured controls & $0.04(0.02-0.079)$ & $0.07(0.05-0.07)$ & $0.05(0.04-0.11)$ \\
\hline $\mathrm{SCl}$ without spasticity & $0.02(0.02-0.03)$ & $0.05(0.04-0.07)$ & $0.04(0.04-0.08)$ \\
\hline SCI with spasticity & $0.08(0.03-0.14)$ & $0.12(0.07-0.31)$ & $0.17(0.14-0.36) \#$ \\
\hline
\end{tabular}

Abbreviations: PI-TA CR, Tibialis Anterior (TA) muscle reflex activity evoked following cutaneous stimulation of the plantar surface; SCI, spinal cord injury. ${ }^{*} P<0.05$ compared with the non-injured control group using the Kruskall-Wallis and the Bonferroni post-test. \# $P<0.05$ \#\# $P<0.01$ compared with early latency reflex activity using the Friedman test and the Bonferroni post-test.

Statistical significant data are represented in bold. 
Table 3 Median PI-TA CR activity during rest, ramp and hold phases of controlled plantarflexion

\begin{tabular}{|c|c|c|c|}
\hline PI-TA CR activity (mV.s) & Rest & Ramp & Hold \\
\hline $\mathrm{SCl}$ without spasticity & $0.41(0.26,0.79)$ & $0.11(0.10,0.17)$ & $0.12(0.09,0.35)$ \\
\hline
\end{tabular}

Abbreviations: PI-TA CR, Tibialis Anterior (TA) muscle reflex activity evoked following cutaneous stimulation of the plantar surface; SCl, spinal cord injury. Data are expressed as median values (25th-75th percentiles). ${ }^{*} P<0.05$ compared with $\mathrm{SCl}$ without spasticity; \#P<0.05 compared with non-injured controls (Kruskall-Wallis test with the Bonferroni post hoc correction). Statistical significant data are represented in bold.

Table 4 Median integrated PI-TA CR activity without background EMG activity analysed during ramp and hold phases of controlled plantarflexion and normalised to the rest period

\begin{tabular}{lcc}
\hline $\begin{array}{l}\text { PI-TA CR activity ((ramp or } \\
\text { hold phase/rest phase)-1) }\end{array}$ & Ramp & Hold \\
\hline Non-inj. contr. & $-0.67(-2.05,-0.12)^{*}$ & $-0.80(-1.83,-0.54)^{* *}$ \\
SCI without sp. & $-0.86(-0.98,-0.68)$ & $-0.90(-0.99,-0.37)^{*}$ \\
SCl with sp. & $-0.16(-0.69,+1.19)$ & $-0.33(-0.79,+0.83)$
\end{tabular}

Abbreviations: Non-Inj., non-injured; PI-TA CR, Tibialis Anterior (TA) muscle reflex activity evoked following cutaneous stimulation of the plantar surface; $\mathrm{SCl}$, spinal cord injury; $\mathrm{Sp}$, spasticity syndrome. Median (25th percentile 75 th percentile). Negative values correspond to $\mathrm{CR}$ inhibition. ${ }^{*} P<0.05$ and ${ }^{* *} P<0.01$ when compared with the rest period (Friedman test with the Bonferroni post hoc correction). Statistical significant data are represented in bold.

TA EMG was statistically similar during either the ramp or hold phases of plantarflexion in subjects with (ramp: 0.11, 0.08-0.13 mV.s, hold: $0.11,0.11-0.16 \mathrm{mV} . \mathrm{s}$ ) or without this syndrome (ramp: 0.09, 0.07-0.12 mV.s, hold: 0.09, 0.06-0.09 mV.s). The inverse relationship between Pl-TA CR activity and plantarflexion torque was not identified in either the non-injured control group or in the SCI group without the spasticity syndrome.

In the group diagnosed with the spasticity syndrome, significant correlations were identified between time after injury and Pl-TA CR activity measured either during the $\operatorname{ramp}(\rho=0.72, P=0.02$, Figure $2 \mathrm{~b})$ or the hold phase of plantarflexion torque $(\rho=0.66$, $P=0.04$ ). Analysis of the normalised Pl-TA CR amplitude (Figure 2c) indicated a reversal from reflex inhibition to facilitation during plantarflexion at approximately 5-6 months after injury, assessed either during the ramp $(\rho=0.79, P=0.01$, Figure $2 \mathrm{c})$ or the hold $(\rho=0.66, \quad P=0.04)$ phases of plantarflexion. In contrast, no relationship was identified between time after injury and Pl-TA CR amplitude in the SCI group without the spasticity syndrome (rest $\rho=0.15, P=0.72$; ramp $\rho=0.63, P=0.10$; hold $\rho=0.26, P=0.54$ ).

Spearman's correlational analysis failed to reveal a clear relationship between Pl-TA CR activity and either the modified Ashworth scale or Penn spasm scores during the rest period or during the ramp and hold phases of plantarflexion torque.

\section{DISCUSSION}

This study is novel in characterising the loss of Pl-TA CR modulation during controlled isometric plantarflexion in subjects with SCI spasticity syndrome and supports a previous study that also identified the loss of medium latency reflex function during gait. ${ }^{11}$ Measurement of Pl-TA reflex activity also provides information regarding the temporal development of CR dysfunction from 5 to 6 months after SCI. At the experimental level, we expect that this method of quantifying loss of physiological inhibition of CR activity during controlled muscle activation after early SCI will add to other reflex diagnostic methods ${ }^{40,41}$ and together will serve to develop antispastic and/or neurorehabilitatory techniques that will better treat the SCI spasticity syndrome, ${ }^{42}$ while maintaining residual motor function. ${ }^{41}$

Loss of segmental and descending Pl-TA CR modulation after SCI Several candidate pathophysiological mechanisms could mediate the loss of physiological inhibition of the Pl-TA CR observed during controlled isometric plantarflexion in subjects with the SCI spasticity syndrome. ${ }^{43}$ At the spinal level, dysfunction of spinal inhibitory mechanisms mediated by gamma-aminobutyric acid is related to cutaneous hyperreflexia after SCI, ${ }^{44,45}$ whereas other structural spinal changes are associated with reflex dysfunction as a consequence of the loss of supraspinal descending control mechanisms. ${ }^{21}$ Damage to descending supraspinal pathways, ${ }^{20,46}$ including the corticospinal ${ }^{47,48}$ or extrapyramidal control systems, ${ }^{19,46,49}$ also may contribute specifically to the change in $\mathrm{CR}$ modulation observed during controlled plantarflexion. Indeed, higher long-latency (200-300 ms) reflex activity observed in the SCI spasticity group during controlled plantarflexion suggests in part that a loss of supraspinal modulation may be implicated as an underlying mechanism. ${ }^{48}$ Nevertheless, another recent study has shown long-latency CR activity in subjects with both complete and incomplete SCI, suggesting that the pathophysiological mechanism mediating this effect could be organised in part at the spinal level. ${ }^{14,50}$

\section{Abnormal Pl-TA CR activity as a sign of the SCI spasticity syndrome}

The SCI spasticity syndrome continues to be defined ${ }^{1}$ and diagnosed on the basis of the detection of muscle hypertonia using the Ashworth scale. ${ }^{34}$ However, this sign represents only one aspect of this motor disorder. ${ }^{2}$ Furthermore, although exaggerated stretch and $\mathrm{H}$ reflex activity have been detected in the clinic and in an animal model of the spasticity syndrome, ${ }^{6,51}$ these signs are not thought to contribute significantly to movement disorder after SCI. ${ }^{52}$

Lower limb flexor CR excitability has often been reported to increase after SCI measured during passive $e^{4,10,15,29,53}$ or active movement. ${ }^{3,11,26,54}$ Furthermore, CR is actively modulated during passive movement, ${ }^{55}$ balance ${ }^{56}$ and gait. ${ }^{26,39,56}$ As such, it is surprising that only one study has observed abnormal CR activity in subjects with the spasticity syndrome during gait after incomplete SCI. ${ }^{11}$

Higher Pl-TA CR activity in subjects diagnosed specifically with the SCI spasticity syndrome, detected without a change in background TA EMG activity during controlled ramp and hold phases of plantarflexion, supports further characterisation of cutaneous hyperreflexia as a test of spasticity for this pathology. Furthermore, the correlation of Pl-TA CR activity with time during subacute and chronic SCI based on the small number of subjects recruited in this study, documented in previous studies, ${ }^{4,14,57}$ also suggests that further characterisation of this reflex measure will provide further information regarding loss of segmental or descending modulatory mechanisms during the late subacute and chronic SCI. 
a
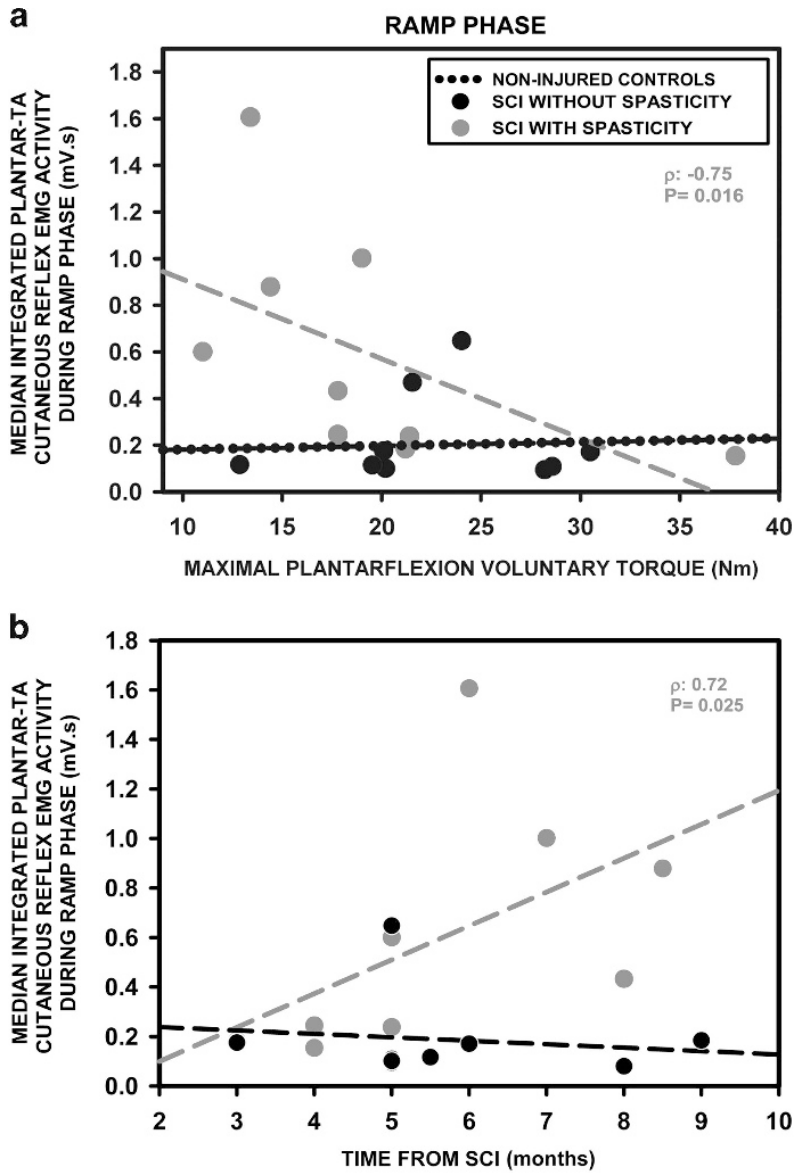

c

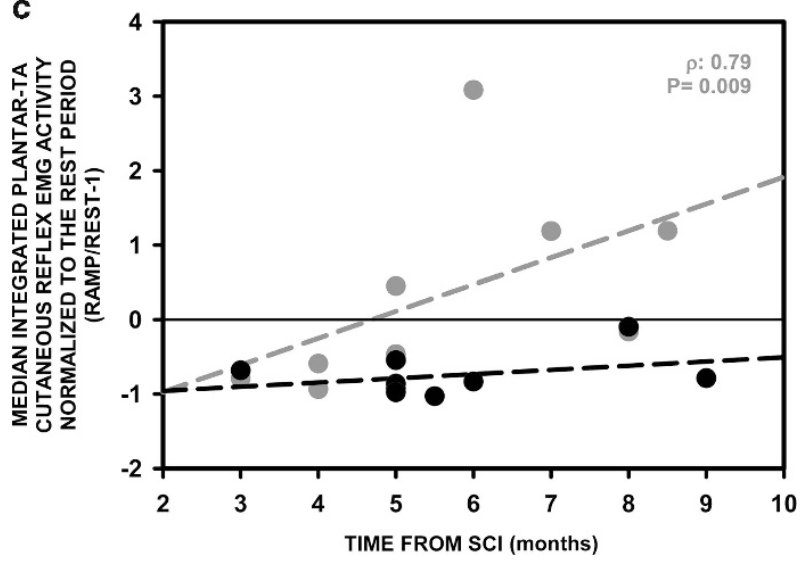

Figure 2 Relationship between plantar-TA reflex activity, maximal TS torque during plantarflexion and time after SCl. PI-TA CR activity evoked following cutaneous electrical stimulation of the plantar sole during controlled plantarflexion in subjects diagnosed with (broken grey lines) or without lower limb spasticity syndrome (broken black lines), plotted against (a) maximal plantarflexion torque and ( $\mathbf{b}$ and $\mathbf{c}$ ) time after $\mathrm{SCl}$. The horizontal dotted line represents the median PI-TA CR activity recorded in the non-injured healthy group.

\footnotetext{
Abnormal Pl-TA CR and lower limb muscle function after SCI A recent study suggests that the development of CR activity following SCI is characteristic of the loss of muscle function during training, ${ }^{14}$ which is tentatively supported by the observation of TS paresis in subjects diagnosed with greater Pl-TA CR activity. Moreover, we have recently demonstrated low intramuscular TA muscle coherence in
}

subjects with the SCI spasticity syndrome, suggesting that this motor disorder is related to a dysfunction of descending motor drive. ${ }^{36} \mathrm{We}$ have also shown that exaggerated lower limb flexor reflex activity evoked in patients with incomplete SCI correlates with poor residual gait function. ${ }^{16}$ However, it is also possible that abnormal CR activity after SCI may be associated with recovery of motor or locomotor function after injury. ${ }^{5-60}$ The relationship between the grade of abnormal CR activity as a factor that either promotes or impedes residual motor function during SCI rehabilitation needs to be addressed in relation to the spasticity syndrome.

Pharmacological treatment for the SCI spasticity syndrome is also known to affect residual motor function after SCI. ${ }^{41,61-63}$ Specifically, the alpha-2 adrenoceptor agonist clonidine has been shown not only to restore inhibition of CR activity after SCI but also to facilitate TA muscle activation during gait, ${ }^{64}$ supporting the hypothesis that adequate $\mathrm{CR}$ control improves residual motor function. We believe that careful CR testing should be used to benchmark standard and new pharmacological strategies for the control of the SCI spasticity syndrome, ${ }^{63,65,66}$ while examining the wider effect of these therapies on residual muscle function after $\mathrm{SCI}^{40,41}$

\section{Limitations and future studies}

No correlation was identified between Pl-TA CR activity and the grade of hypertonia or spasm frequency, perhaps reflecting the poor sensitivity of the modified Ashworth and Penn scales to detect flexor reflex activity. Measurement of spasm activity in clinical studies is usually performed with the Penn scale, ${ }^{35}$ but this scale often fails to correlate with the underlying pathophysiological mechanisms of SCI spasticity syndrome during movement, ${ }^{7}$ or indeed with CR activity in general. ${ }^{29,31}$ Adoption of clinically relevant outcome measures, such as the Spinal Cord Assessment Tool for Spastic reflexes (SCATS), which provides a better evaluation of evoked flexor reflex activity, should also be evaluated. ${ }^{16,36,67}$

Several experimental aspects of the reflex testing technique remain to be addressed, such as identifying correctly CR thresholds and normalising EMG data across experimental groups. We defined TA CR reflex threshold in part on the subjective assessment of the stimulus being innocuous. We recommend the use of a systematic method to identify reflex threshold such as the method of limits. However, perception of the stimulus may be affected in those subjects with greater SCI severity and would therefore require reflex threshold identification based on the TA reflex response alone. It is important to note that the reflex threshold method used in this study demonstrated no significant differences between experimental groups. With respect to normalising EMG data across experimental groups, the TA CR response was normalised for all subjects by recording activity measured during rest and at 50\% maximal voluntary torque. This sub-maximal isometric normalisation method has been used in other biomechanical studies and provides good reliability, ${ }^{68,69}$ and this may be optimal for subjects who are not able to generate high maximal contractions.

Finally, the contribution of TA muscle coactivation during TS muscle contraction should be assessed, as this may account for the correlation between plantarflexor weakness identified in those subjects with Pl-TA hyperreflexia. However, in a previous study, we demonstrated that TA EMG activity measured during ramp plantarflexion was the same in subjects with or without SCI spasticity syndrome. ${ }^{37}$ Examination of TA, Soleus and Gastrocnemius muscle activity during controlled plantarflexion will be instrumental in assessing the role of abnormal CR activity in TS muscle weakness, by controlling for possible lower limb muscle coactivation. 


\section{CONCLUSION}

This study not only adds to the previous observation of loss of CR inhibition during gait in subjects with $\mathrm{SCI}^{11}$ but also demonstrates that measurement of the loss of physiological inhibition of CR activity during controlled isometric plantarflexion is characteristic of the SCI spasticity syndrome. The reflex testing procedure developed here provides a useful technique to differentiate abnormal CR activity in subjects with clinical signs of SCI spasticity and could be used to benchmark novel pharmacological or sensorimotor rehabilitation techniques designed to re-establish appropriate inhibitory modulation of CR function, thereby contributing to the better management of SCI spasticity. ${ }^{40,42,66,70}$

\section{DATA ARCHIVING}

There were no data to deposit.

\section{CONFLICT OF INTEREST}

The authors declare no conflict of interest.

\section{ACKNOWLEDGEMENTS}

We are grateful to Professor Volker Dietz for constructive critical comments and to Dr Ksenija Jovanovic for proofreading the manuscript. We are also grateful for the continued support of Dr Ana Esclarín, Angel Gil and the medical staff of the 'Servicio de Rehabilitación' and for the participation of the physiotherapists at the 'Hospital Nacional de Parapléjicos' in recruiting subjects with subacute spinal cord injury. This project was funded by the 'Consejería de Sanidad de Castilla-La Mancha' (03019-00, EQ02015), 'Fundación Mutua Madrileña 2013' and the 'Consolider-Ingenio 2010 Hyper' from the 'Ministerio de Ciencia e Innovación' (CSDOOC-09-61313 to EBE).

1 Lance JW. The control of muscle tone, reflexes, and movement: Robert Wartenberg Lecture. Neurology 1980; 30: 1303-1313.

2 Katz RT, Rymer WZ. Spastic hypertonia: mechanisms and measurement. Arch Phys Med Rehabil 1989; 70: 144-155.

3 Stein RB, Yang JF, Belanger M, Pearson KG. Modification of reflexes in normal and abnormal movements. Prog Brain Res 1993; 97: 189-196.

4 Hiersemenzel LP, Curt A, Dietz V. From spinal shock to spasticity: neuronal adaptations to a spinal cord injury. Neurology 2000; 54: 1574-1582.

5 Bennett DJ. Demystifying spasticity: reply to Dietz. J Neurophysiol 2008; 99 1041-1043.

6 Taylor JS, Friedman RF, Munson JB, Vierck CJ Jr. Stretch hyperreflexia of triceps surae muscles in the conscious cat after dorsolateral spinal lesions. J Neurosci 1997; 17: 5004-5015

7 Dietz V, Sinkjaer T. Spastic movement disorder: impaired reflex function and altered muscle mechanics. Lancet Neurol 2007; 6: 725-733.

8 Gorassini MA, Knash ME, Harvey PJ, Bennett DJ, Yang JF. Role of motoneurons in the generation of muscle spasms after spinal cord injury. Brain 2004; 127: 2247-2258.

9 Wu M, Hornby TG, Kahn JH, Schmit BD. Flexor reflex responses triggered by imposed knee extension in chronic human spinal cord injury. Exp Brain Res 2006; 168: 566-576.

10 Dimitrijevic MR, Nathan PW. Studies of spasticity in man. 3. Analysis of revlex activity evoked by noxious cutaneous stimulation. Brain 1968; 91: 349-368.

11 Jones CA, Yang JF. Reflex behavior during walking in incomplete spinal-cord-injured subjects. Exp Neurol 1994; 128: 239-248.

12 Shahani BT, Young RR. Human flexor reflexes. J Neurol Neurosurg Psychiatry 1971; 34: 616-627.

13 Anderson DK, Beattie M, Blesch A, Bresnahan J, Bunge M, Dietrich D et al. Recommended guidelines for studies of human subjects with spinal cord injury. Spinal Cord 2005; 43: 453-458.

14 Dietz V, Grillner S, Trepp A, Hubli M, Bolliger M. Changes in spinal reflex and locomotor activity after a complete spinal cord injury: a common mechanism? Brain 2009; 132: 2196-2205.

15 Hornby TG, Rymer WZ, Benz EN, Schmit BD. Windup of flexion reflexes in chronic human spinal cord injury: a marker for neuronal plateau potentials? J Neurophysiol 2003; 89: 416-426.

16 Bravo-Esteban E, Taylor J, Abian-Vicen J, Albu S, Simon-Martinez C, Torricelli D et al. Impact of specific symptoms of spasticity on voluntary lower limb muscle function, gait and daily activities during subacute and chronic spinal cord injury. Neurorehabilitation 2013; 33: 531-543.
17 Little JW, Micklesen P, Umlauf R, Britell C. Lower extremity manifestations of spasticity in chronic spinal cord injury. Am J Phys Med Rehabil 1989; 68: 32-36.

18 Clarke RW, Ford TW, Taylor JS. Reflex actions of selective stimulation of sural nerve C fibres in the rabbit. Q J. Exp Physiol 1989; 74: 681-690.

19 Clarke RW, Eves S, Harris J, Peachey JE, Stuart E. Interactions between cutaneous afferent inputs to a withdrawal reflex in the decerebrated rabbit and their control by descending and segmental systems. Neuroscience 2002; 112: 555-571.

20 Jenner JR, Stephens JA. Cutaneous reflex responses and their central nervous pathways studied in man. J Physiol 1982; 333: 405-419.

21 Beauparlant J, van den Brand R, Barraud Q, Friedli L, Musienko P, Dietz V et al. Undirected compensatory plasticity contributes to neuronal dysfunction after severe spinal cord injury. Brain 2013; 136: 3347-3361.

22 Nakajima T, Sakamoto M, Tazoe T, Endoh T, Komiyama T. Location specificity of plantar cutaneous reflexes involving lower limb muscles in humans. Exp Brain Res 2006; 175: 514-525.

23 Hagbarth KE. Excitatory and inhibitory skin areas for flexor and extensor motoneurons. Acta Physiol Scand Suppl 1952; 26: 1-58.

24 Sonnenborg FA, Andersen OK, Arendt-Nielsen L. Modular organization of excitatory and inhibitory reflex receptive fields elicited by electrical stimulation of the foot sole in man. Clin Neurophysiol 2000; 111: 2160-2169.

25 Yang JF, Stein RB. Phase-dependent reflex reversal in human leg muscles during walking. J Neurophysiol 1990; 63: 1109-1117.

26 Knikou M. Plantar cutaneous input modulates differently spinal reflexes in subjects with intact and injured spinal cord. Spinal Cord 2007; 45: 69-77.

27 Knikou M. Plantar cutaneous afferents normalize the reflex modulation patterns during stepping in chronic human spinal cord injury. J Neurophysiol 2010; 103: 1304-1314.

28 Katz RT, Rovai GP, Brait C, Rymer WZ. Objective quantification of spastic hypertonia: correlation with clinical findings. Arch Phys Med Rehabil 1992; 73: 339-347.

29 Parise M, Garcia-Larrea L, Mertens P, Sindou M, Mauguiere F. Clinical use of polysynaptic flexion reflexes in the management of spasticity with intrathecal baclofen. Electroencephalogr Clin Neurophysiol 1997; 105: 141-148.

30 Penn RD, Savoy SM, Corcos D, Latash M, Gottlieb G, Parke B et al. Intrathecal baclofen for severe spinal spasticity. N Engl J Med 1989; 320: 1517-1521.

31 Priebe MM, Sherwood AM, Thornby JI, Kharas NF, Markowski J. Clinical assessment of spasticity in spinal cord injury: a multidimensional problem. Arch Phys Med Rehabil 1996; 77: 713-716.

32 Maynard FM Jr., Bracken MB, Creasey G, Ditunno JF Jr., Donovan WH, Ducker TB et al. International Standards for Neurological and Functional Classification of Spinal Cord Injury. American Spinal Injury Association. Spinal Cord 1997; 35: 266-274.

33 Medical Research Council. Aids to the examination of the peripheral nervous system. Memorandum no. 45. Her Majesty's Stationery Office, London, 1981.

34 Bohannon RW, Smith MB. Interrater reliability of a modified Ashworth scale of muscle spasticity. Phys Ther 1987; 67: 206-207.

35 Penn RD, Kroin JS. Long-term intrathecal baclofen infusion for treatment of spasticity. J Neurosurg 1987; 66: 181-185.

36 Bravo-Esteban E, Taylor J, Aleixandre M, Simon-Martinez C, Torricelli D, Pons JL et al. Tibialis Anterior muscle coherence during controlled voluntary activation in patients with spinal cord injury: diagnostic potential for muscle strength, gait and spasticity. J Neuroeng Rehabil 2014; 11: 23.

37 Gómez-Soriano J, Castellote JM, Pérez-Rizo E, Esclarin A, Taylor JS. Voluntary ankle flexor activity and adaptive coactivation gain is decreased by spasticity during subacute spinal cord injury. Exp Neurol 2010; 224: 507-516.

38 Baken BC, Dietz V, Duysens J. Phase-dependent modulation of short latency cutaneous reflexes during walking in man. Brain Res 2005; 1031: 268-275.

39 Van Wezel BM, Ottenhoff FA, Duysens J. Dynamic control of location-specific information in tactile cutaneous reflexes from the foot during human walking. J Neurosci 1997; 17: 3804-3814.

40 Chu VW, Hornby TG, Schmit BD. Effect of antispastic drugs on motor reflexes and voluntary muscle contraction in incomplete spinal cord injury. Arch Phys Med Rehabil 2014; 95: 622-632

41 Theiss RD, Hornby TG, Rymer WZ, Schmit BD. Riluzole decreases flexion withdrawal reflex but not voluntary ankle torque in human chronic spinal cord injury. J Neurophysiol 2011; 105: 2781-2790.

42 Gómez-Soriano J, Taylor J. Cutaneous lower limb hyperreflexia in patients with spinal cord injury spasticity: Modulation with vibration. Physiotherapy 2011, 97(Suppl 1): eS419 (abstract 207).

43 Biering-Sorensen F, Nielsen JB, Klinge K. Spasticity-assessment: a review. Spinal Cord 2006; 44: 708-722.

44 Kakinohana O, Hefferan MP, Nakamura S, Kakinohana M, Galik J, Tomori Z et al. Development of GABA-sensitive spasticity and rigidity in rats after transient spinal cord ischemia: a qualitative and quantitative electrophysiological and histopathological study. Neuroscience 2006; 141: 1569-1583.

45 Meisner JG, Marsh AD, Marsh DR. Loss of GABAergic interneurons in laminae I-III of the spinal cord dorsal horn contributes to reduced GABAergic tone and neuropathic pain after spinal cord injury. J Neurotrauma 2010; 27: 729-737.

46 Clarke RW, Ford TW, Taylor JS. Adrenergic and opioidergic modulation of a spinal reflex in the decerebrated rabbit. J Physiol 1988; 404: 407-417.

47 Gibbs J, Harrison LM, Stephens JA. Cutaneomuscular reflexes recorded from the lower limb in man during different tasks. JPhysiol 1995; 487: 237-242.

48 Nielsen J, Petersen N, Fedirchuk B. Evidence suggesting a transcortical pathway from cutaneous foot afferents to tibialis anterior motoneurones in man. JPhysiol 1997; 501: 473-484. 
49 Clarke RW, Harris J, Houghton AK. Spinal 5-HT-receptors and tonic modulation of transmission through a withdrawal reflex pathway in the decerebrated rabbit. Br J Pharmacol 1996; 119: 1167-1176.

50 Hubli M, Bolliger M, Dietz V. Neuronal dysfunction in chronic spinal cord injury. Spinal Cord 2011; 49: 582-587.

51 Nielsen JB, Crone C, Hultborn H. The spinal pathophysiology of spasticity-from a basic science point of view. Acta Physiol (Oxf) 2007; 189: 171-180.

52 Dietz V. Spastic movement disorder. Spinal Cord 2000; 38: 389-393.

53 Roby-Brami A, Bussel B. Long-latency spinal reflex in man after flexor reflex afferent stimulation. Brain 1987; 110: 707-725.

54 Duysens J, Baken BC, Burgers L, Plat FM, Den Otter AR, Kremer HP. Cutaneous reflexes from the foot during gait in hereditary spastic paraparesis. Clin Neurophysiol 2004; 115: 1057-1062.

55 Nakajima T, Kamibayashi K, Takahashi M, Komiyama T, Nakazawa K. Phase-dependent modulation of cutaneous reflexes in tibialis anterior muscle during passive stepping. Neurol Res 2008; 30: 46-51.

56 Zehr EP, Stein RB. What functions do reflexes serve during human locomotion? Prog Neurobiol 1999; 58: 185-205.

57 Norton JA, Bennett DJ, Knash ME, Murray KC, Gorassini MA. Changes in sensoryevoked synaptic activation of motoneurons after spinal cord injury in man. Brain 2008; 131: 1478-1491.

58 Bussel B, Roby-Brami A, Yakovleff A, Bennis N. Late flexion reflex in paraplegic patients. Evidence for a spinal stepping generator. Brain Res Bull 1989; 22: 53-56.

59 Nicol DJ, Granat MH, Baxendale RH, Tuson SJ. Evidence for a human spinal stepping generator. Brain Res 1995; 684: 230-232.

60 Nadeau S, Jacquemin G, Fournier C, Lamarre Y, Rossignol S. Spontaneous motor rhythms of the back and legs in a patient with a complete spinal cord transection. Neurorehabil Neural Repair 2010; 24: 377-383.
61 Thomas CK, Hager-Ross CK, Klein CS. Effects of baclofen on motor units paralysed by chronic cervical spinal cord injury. Brain 2010; 133: 117-125.

62 Angeli CA, Edgerton VR, Gerasimenko YP, Harkema SJ. Altering spinal cord excitability enables voluntary movements after chronic complete paralysis in humans. Brain 2014; 137: 1394-1409.

63 Gómez-Soriano J, Goiriena E, Taylor J. Spasticity therapy reacts to astrocyte GluA1 receptor upregulation following spinal cord injury. Br J Pharmacol 2010; 161: 972-975.

64 Remy-Neris O, Barbeau H, Daniel O, Boiteau F, Bussel B. Effects of intrathecal clonidine injection on spinal reflexes and human locomotion in incomplete paraplegic subjects. Exp Brain Res 1999; 129: 433-440.

65 Mirbagheri MM, Chen D, Rymer WZ. Quantification of the effects of an alpha-2 adrenergic agonist on reflex properties in spinal cord injury using a system identification technique. J Neuroeng Rehabil 2010; 7: 29.

66 Ávila-Martín G, Galán-Arriero I, Gómez-Soriano J, Taylor J. Treatment of rat spinal cord injury with the neurotrophic factor albumin-oleic acid: translational application for paralysis, spasticity and pain. Plos ONE 2011; 6: e26107.

67 Benz EN, Hornby TG, Bode RK, Scheidt RA, Schmit BD. A physiologically based clinical measure for spastic reflexes in spinal cord injury. Arch Phys Med Rehabil 2005; 86: 52-59.

68 Murley GS, Menz HB, Landorf KB, Bird AR. Reliability of lower limb electromyography during overground walking: a comparison of maximal- and sub-maximal normalisation techniques. J Biomech 2009; 43: 749-756.

69 Ball N, Scurr J. An assessment of the reliability and standardisation of tests used to elicit reference muscular actions for electromyographical normalisation. J Electromyogr Kinesiol 2010; 20: 81-88.

70 Tillakaratne NJ, de Leon RD, Hoang TX, Roy RR, Edgerton VR, Tobin AJ. Use-dependent modulation of inhibitory capacity in the feline lumbar spinal cord. J Neurosci 2002; 22: 3130-3143. 